\title{
Cell Type-Specific Firing during Ripple Oscillations in the Hippocampal Formation of Humans
}

\author{
Michel Le Van Quyen, ${ }^{1,2}$ Anatol Bragin, ${ }^{3}$ Richard Staba, ${ }^{3}$ Benoit Crépon, ${ }^{1,2}$ Charles L. Wilson, ${ }^{3}$ and Jerome Engel Jr ${ }^{3}$ \\ ${ }^{1}$ Centre National de la Recherche Scientifique, Cognitive Neuroscience and Brain Imaging Laboratory, Unité Propre de Recherche 640, Hôpital de la Pitié- \\ Salpêtrière, 75651 Paris, France, ${ }^{2}$ Université Pierre et Marie Curie-Paris 6, 75005 Paris, France, and ${ }^{3}$ Neurology Department, David Geffen School of \\ Medicine at University of California, Los Angeles, Los Angeles, California 90095
}

\begin{abstract}
High-frequency field ripples occur in the rodent hippocampal formation and are assumed to depend on interneuron type-specific firing patterns, structuring the activity of pyramidal cells. Ripples with similar characteristics are also present in humans, yet their underlying cellular correlates are still unknown. By in vivo recording interneurons and pyramidal cells in the human hippocampal formation, we find that cell type-specific firing patterns and phase-locking on a millisecond timescale can be distinguished during ripples. In particular, pyramidal cells fired preferentially at the highest amplitude of the ripple, but interneurons began to discharge earlier than pyramidal cells. Furthermore, a large fraction of cells were phase-locked to the ripple cycle, but the preferred phase of discharge of interneurons followed the maximum discharge probability of pyramidal neurons. These relationships between human ripples and unit activity are qualitatively similar to that observed in vivo in the rodents, suggesting that their underlying mechanisms are similar.
\end{abstract}

Key words: ripple; sharp wave; oscillation; interneuron; human hippocampus; EEG

\section{Introduction}

Sharp-wave ripple oscillations $(120-200 \mathrm{~Hz})$ occur in rodent hippocampal-entorhinal networks during immobility or slowwave sleep (O’Keefe and Nadel, 1978; Buzsáki et al., 1992, 2003; Chrobak et al., 2000). These ripples originate from the synchronized firing of CA3 cells and spread downstream as spatially coherent oscillations along the CA1-subicular-entorhinal axis (Chrobak and Buzsáki, 1996). This coactivation of hippocampal and neocortical pathways are thought to be crucial for memory consolidation processes, during which memories are gradually translated from short-term hippocampal to longer-term neocortical stores (Buzsáki, 1989; Lee and Wilson, 1992; Wilson and McNaughton, 1994; Behrens et al., 2005).

On the cellular level, ripples reflect the summed activity of pyramidal cells and interneurons coupled to each oscillatory cycle. In support of this, recent work has identified that both pyramidal neurons and various classes of interneurons (e.g., basket cells and bistratified cells) display their highest discharge probability around the negative peak of ripple cycle (Ylinen et al., 1995; Csicsvari et al., 1999, 2000; Klausberger et al., 2003, 2004). Furthermore, other classes of GABAergic interneurons fire at distinct

Received Jan. 31, 2008; revised March 26, 2008; accepted April 6, 2008.

This work was supported by National Institutes of Health Grants NS-33310 and NS-02808 and by European Union-FP7 Project EPILEPSIAE (Evolving Platform for Improving Living Expectation of Patients Suffering from IctAI Events).

Correspondence should be addressed to either of the following: Michel Le Van Quyen, Centre National de la Recherche Scientifique, Cognitive Neuroscience and Brain Imaging Laboratory, Unité Propre de Recherche 640, Hôpital de la Pitié-Salpêtrière, 47 Boulevard de I'Hôpital, 75651 Paris cedex 13, France, E-mail: lenalm@ext.jussieu.fr; or Anatol Bragin, Neurology Department, David Geffen School of Medicine at University of California, Los Angeles, 710, Westwood Plaza, Los Angeles, CA 90095, E-mail: ABragin@mednet.ucla.edu.

D0I:10.1523/JNEUROSCI.0437-08.2008

Copyright $\odot 2008$ Society for Neuroscience $\quad$ 0270-6474/08/286104-07\$15.00/0 time points during a given oscillation (Klausberger et al., 2003, 2004). For example, oriens-lacunosum moleculare interneurons are primarily silent during the ripples, whereas axo-axonic interneurons slightly increased their firing activity at the beginning of the ripples but became silent after the highest amplitude of the ripple episode. As different classes of interneurons innervate distinct domains of pyramidal cells, these differential firing dynamics suggest that diverse interneuronal populations regulate the synchronization of pyramidal cells during a given ripple (Somogyi and Klausberger, 2005).

Are these hippocampal discharge patterns universal across all mammalian species, including humans? In monkeys, data from hippocampal recordings seem to present a qualitatively similar picture to that seen in rodents (Skaggs et al., 2007). Ripples with similar characteristics were also recorded in human hippocampus and entorhinal cortex (Bragin et al., 1999, 2002a; Staba et al., 2002, 2004; Clemens et al., 2007). Nevertheless, it is still unknown whether the cellular mechanisms of these oscillations are the same. The goal of this study is to characterize the electrophysiological features of single neurons recorded during ripple oscillations within the medial temporal lobes in humans. We report that distinct classes of firing pattern and phase-related firing can be distinguished during sharp wave-associated high-frequency ripples, suggesting a similar cellular mechanism to ripples in rodents.

\section{Materials and Methods}

Patients. The subjects were 11 patients with pharmacologically intractable epilepsy, who had been implanted with depth electrodes to localize the focus of seizure onset. Extensive noninvasive monitoring did not yield concordant data corresponding to a single resectable epileptogenic focus. Therefore, the patients were implanted with chronic depth elec- 
trodes for 7-10 d to determine the seizure focus for possible surgical resection. For each patient, the placement of the depth electrodes, in combination with microwires, was determined exclusively by clinical criteria (Fried et al., 1999). All studies conformed to the guidelines of the Medical Institutional Review Board at University of California, Los Angeles.

Recordings. Each depth electrode probe had a total of nine microwires at its end, eight active recording channels, and one reference. For six patients, each microwire was sampled at $10 \mathrm{kHz}$ with 12-bit precision (EGAA Acquisition System; RC Electronics, Santa Barbara, CA) and for five other patients with a sampling rate of $28-32 \mathrm{kHz}$ (Cheetah Recording System; Neuralynx, Tucson, AZ). We selected the bundles of microwires located in the hippocampus and entorhinal cortex determined on the basis of postimplant computed tomography scans coregistered with preimplant $1.5 \mathrm{~T}$ magnetic resonance imaging scans. Separated by $500 \mu \mathrm{m}$, the electrodes record nonoverlapping cell populations. All patients had bilaterally implanted depth electrodes in multiple sites to contrast rival hypotheses about the location of the epileptogenic zone. Therefore, only a portion of the total recording electrodes included contacts in regions where seizures were thought to originate. The patients were awake, lying in bed with their eyes opened, or asleep (episodes of nonrapid eye movement sleep, confirmed by two EEG channels, two electro-ocular channels to monitor eye movements, and two electromyogram channels recording from the patient's chin).

Spike sorting. All channels were high-pass filtered at $300 \mathrm{~Hz}$ and were visually examined for the presence of unit activities. In those microwires with clear unit activities, we performed spike detection ( $>4: 1$ signal-tonoise ratio) and spikes sorting using KlustaKwik 1.5 program (Harris et al., 2001) (software available at http://klustakwik.sourceforge.net/), which uses the five principal components of the spike shape and an unsupervised conditional expectation maximization clustering algorithm (Hazan et al., 2006). After automatic clustering, the clusters containing nonspike waveforms were visually deleted, and then the units were further isolated using a manual cluster cutting method. Only units with clear boundaries and $<0.5 \%$ of spike intervals within a $1 \mathrm{~ms}$ refractory period are included in the present analysis. Typically, we isolated one or two distinct neurons from each microwire, but in several cases, we observed up to four distinct neurons from a single microwire.

Ripple detection. Channels demonstrating oscillations with largeamplitude sinusoid-like waves with frequencies between 80 and $200 \mathrm{~Hz}$ that were discernable above background EEG were selected for analysis. An automatic detection of ripple episodes was then achieved as described previously (Staba et al., 2004). In brief, an automated detection to identify the periods within the signal exhibits high-power oscillatory activity, longer than a minimal duration. The duration threshold was set to $20 \mathrm{~ms}$ (i.e., two to three cycles of ripple oscillations), and the amplitude threshold was set to three SDs calculated over the entire length of the bandpassfiltered signal $(80-200 \mathrm{~Hz})$. All the traces are then aligned at the beginning of the ripple episode defined, where the bandpass-filtered activities crossed a threshold at three times the SD. The qualitative nature of our results is insensitive to the precise choice of thresholds ( $>3 \mathrm{SD}$ ).

Ripple analysis. A wavelet time-frequency analysis was used to determine precisely the mean frequency, the beginning, highest amplitude, and end of the ripples. The advantage of the wavelet analysis lies in the fact that the time resolution is variable with frequency, so that high frequencies have a sharper time resolution (Le Van Quyen and Bragin, 2007). The Morlet wavelet is applied and uses a wave-like scalable function that is well localized in both time and frequency as follows:

$$
\Psi_{\tau, f}(u)=\sqrt{f} \exp (j 2 \pi f(u-\tau)) \exp \left(-\frac{(u-\tau)^{2}}{\sigma^{2}}\right) .
$$

This wavelet represents the product of a sinusoidal wave at frequency $f$, with a Gaussian function centered at time $\tau$, with a SD $\sigma$ proportional to the inverse of $f$. The wavelet coefficients of a signal $x(t)$ as a function of time $(\tau)$ and frequency $(f)$ are defined as follows:

$$
W(\tau, f)=\int_{-\infty}^{+\infty} x(u) \Psi_{\tau, f}(u) d u,
$$

This depends solely on $\sigma$, which sets the number of cycles of the wavelet: $n c o=6 f \sigma$. This value $n c o$ determines the frequency resolution of the analysis by setting the width of the frequency interval for which phases are measured. We chose $n c o=5$. As a criterion of the significance for the time-frequency representations, we required that the time-frequency peak energy to exceed the mean + three SDs of baseline.

Phase-locking between spikes and field oscillations. To analyze the phase-locking between single units and field oscillations, we used the procedure described by Jacobs et al. (2007). We first downsampled recordings to $2 \mathrm{kHz}$. To minimize the contribution of low-frequency components of spikes toward spectral calculations, we replaced the samples from $2 \mathrm{~ms}$ before to $8 \mathrm{~ms}$ after each spike with a linear interpolation of the underlying field signal. Then, we computed oscillatory phase and power of the field potential using Morlet wavelets $($ nco $=5)$ at frequencies between 30 and $240 \mathrm{~Hz}$. We considered a neuron phase-locked at a particular frequency if the hypothesis of circular uniformity for its field phase distribution could be rejected at $p<0.001$ using a Bonferroni's corrected Rayleigh test (Fisher et al., 1993). Briefly, given $n$ phase values $\phi_{i}$ define the first trigonometric moment as follows:

$$
m=(1 / n) \sum_{1}^{n} e^{j \phi i}
$$

The sample mean direction or preferred phase $\mu$ is given by the orientation of $m$, whereas the mean resultant value $R$ is given by the modulus of $m$. The Rayleigh statistic is $Z=n R^{2}$, and the probability that the null hypothesis of sample uniformity holds is given by $P=e^{-Z}[1+(2 Z-$ $\left.\left.Z^{2}\right) /(4 n)-\left(24 Z-132 Z^{2}+76 Z^{3}-9 Z^{4}\right) /\left(288 n^{2}\right)\right]$. For $n>50$, the approximation $P=e^{-Z}$ is adequate (Fisher et al., 1993; Siapas et al., 2005). All significantly phase-locked units were fit with a von Mises distribution with density as follows:

$$
f(\phi)=\left(1 /\left[2 \pi I_{0}(\kappa)\right]\right) e^{\kappa \cos (\phi-\mu)},
$$

for

$$
-\pi \leq \phi<\pi \text { and } 0 \leq \kappa<\infty .
$$

The estimate of the von Mises mean direction parameter $(\mu)$ gives the preferred phase (Siapas et al., 2005).

\section{Results}

We recorded 41,135 field ripples with microelectrodes chronically implanted in the mesial temporal lobes of 11 subjects during quiet wakefulness or slow-wave sleep (recording durations from 13 to $331 \mathrm{~min}$; total recording time, $33.2 \mathrm{~h}$ ). As in rodents, these high-frequency oscillations were observed in the hippocampus and entorhinal cortex (Fig. 1 A) (Bragin et al., 1999, 2002a; Staba et al., 2002, 2004), usually at the onset of sharp waves $(82 \%)$ [consistent with the findings of Clemens et al. (2007) and Urrestarazu et al. (2007)]. Spectral analyses revealed a frequency peak between 80 and $160 \mathrm{~Hz}$ (maximum of mean peak, $98 \pm 25.7 \mathrm{~Hz}$; mean \pm SD), which is lower in frequency than the $140-200 \mathrm{~Hz}$ ripples of rats (Csicsvari et al., 1999) or the 120-180 Hz ripples of the mouse (Buzsáki et al., 2003) but close to the $100-120 \mathrm{~Hz}$ oscillations observed in monkeys (Skaggs et al., 2007). This is further supported by a peak-to-peak time between ripple troughs of $9.5 \pm 2.2 \mathrm{~ms}(\sim 5 \mathrm{~ms}$ in rodents, respectively) (Chrobak and Buzsáki, 1996). Duration of ripples was $38.0 \pm 33.1 \mathrm{~ms}$, which is comparable with that observed in rodents and monkeys.

A total of 133 single cells were detected and selected for analysis (90 in the entorhinal cortex and 43 in the hippocampus). Consistent with previous studies in rodents (Csicsvari et al., 1999), cells could be divided in two classes of putative pyramidal cells and interneurons on the basis of their average spike duration and burst propensity by the first moment of the autocorrelogram (Fig. $1 B, C$ ). Hartigan's dip statistic (Hartigan and Hartigan, 

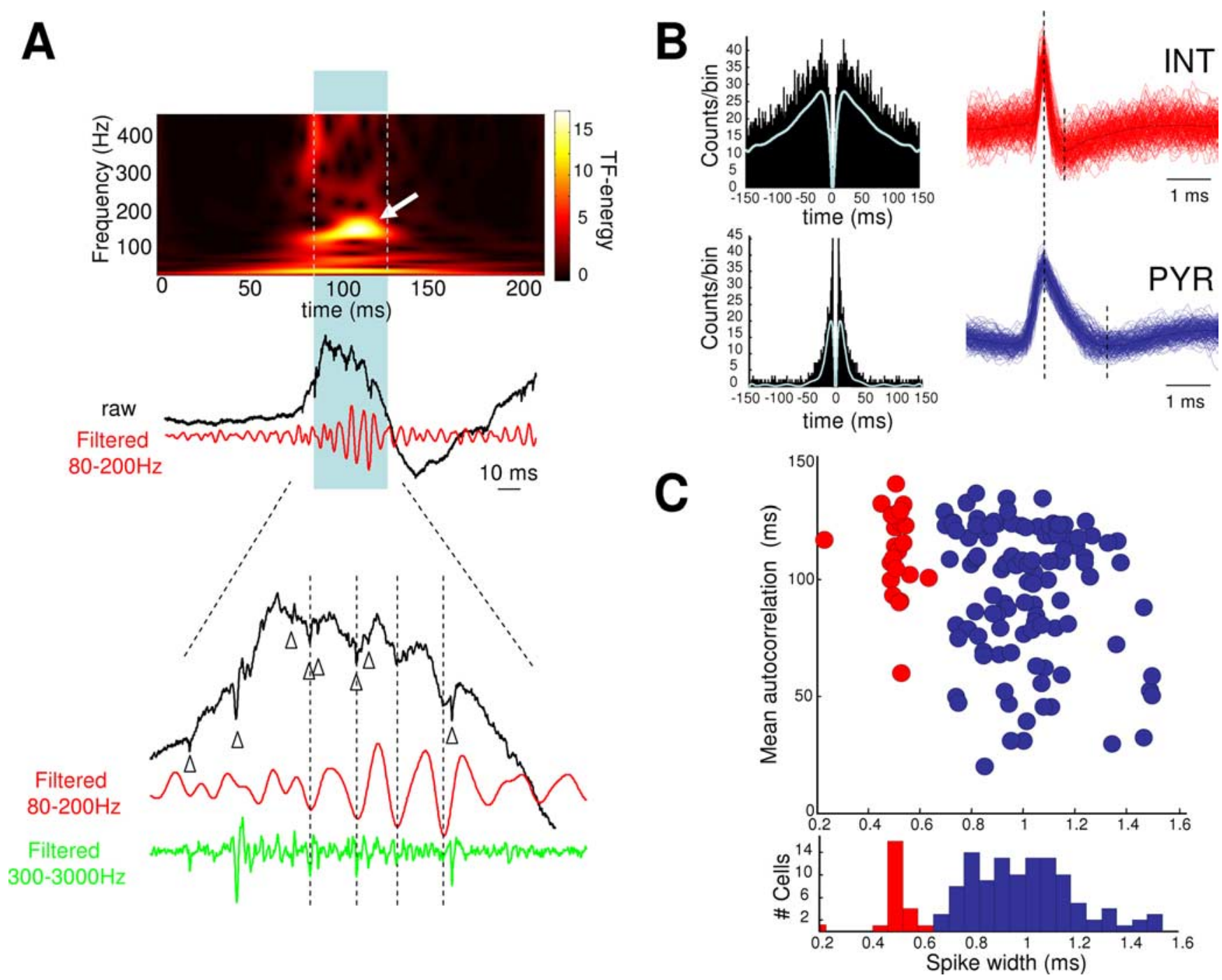

Figure 1. A, Single-field ripple event (black trace) detected in the hippocampus by bandpass filtering (red trace) or by the time-frequency representation (top). The power of the oscillation is depicted by a color scale coded in SDs above the mean power of an event-free baseline. The expanded trace (bottom panel) shows unit activities $(\Delta)$ during the oscillations, mostly occurring around the negative peak of ripple cycle. $\boldsymbol{B}$, Physiological identification of putative pyramidal cells (PYR; blue) and interneurons (INT; red). The spike width (right), measured as the average time between the peak and the trough of the action potential, shows a characteristic difference between pyramidal cell and interneuron. The dotted lines indicate times of peak and trough for each cell. In addition, the autocorrelogram (left) of the spike time also shows that putative pyramidal cells have a peak at $3-5$ ms followed by a rapid exponential decay, whereas interneurons exhibited a much slower decay. C, Top, First moment (i.e., the mean value) of the autocorrelogram as a function of spike width for interneurons (red) and pyramidal cells (blue). Bottom, Histogram of the spike width distribution.

$1985)$ demonstrated that the peak-to-trough width was bimodal $\left(p<10^{-2}\right.$, significance obtained via Monte Carlo simulation with $n=10^{5}$ ) (Mechler and Ringach, 2002) (Fig. 1C, histogram in bottom panel) with a dip at $0.62 \mathrm{~ms}$ and with one group of cells characterized by a narrow waveform $(0.5 \pm 0.07 \mathrm{~ms}$; putative interneurons, $n=23$ ), whereas the second group had a wide waveform $(1.0 \pm 0.19 \mathrm{~ms}$; putative pyramidal cells, $n=110)$. Putative interneurons had an autocorrelogram with a slow decay and a longer first moment. In contrast, pyramidal neurons have often a peak at $3-5 \mathrm{~ms}$ in the autocorrelograms (i.e., a tendency to fire complex-spikes bursts), followed by a fast exponential decay, exhibiting a shorter first moment (Fig. $1 B, C$ ). On average, putative pyramidal cells discharged at a lower rate than interneurons ( $4.6 \pm 8.3$ and $6.8 \pm 6.6 \mathrm{~Hz}$, respectively) but several cells in both groups had overlapping firing properties (range, 0.04-21.7 and $1.4-25.8 \mathrm{~Hz}$, respectively). No regional differences in cell features were found between the hippocampus and entorhinal cortex, or inside and outside of the epileptic zone (Viskontas et al., 2006).
To examine the temporal relationships between ripples and single cells, we aligned individual ripples on their initiation and constructed ripple-triggered spike histograms for each microelectrode using the same time periods.

During ripples in rodents, $>10 \%$ of pyramidal cells discharge synchronously with the oscillating local field potential (Buzsáki et al., 1992; Ylinen et al., 1995; Csicsvari et al., 1999, 2000). In accord with these findings, we found that $29 \%$ of pyramidal neurons (Fig. $2 A i, B$ ) and $22 \%$ of interneurons (Fig. $2 A i i, B$ ) increased their firing rate more than two times during ripples relative to baseline epochs. The mean firing rate for pyramidal cells during ripple oscillations was $16 \pm 19.2 \mathrm{~Hz}$ (baseline, $3.1 \pm 2.1 \mathrm{~Hz}$ ) and for interneurons $19.2 \pm 9.6 \mathrm{~Hz}$ (baseline, $5.1 \pm 2.0 \mathrm{~Hz}$ ). As also observed in rats [termed antisharp wave cells by Csicsvari et al. (1999)], a small percentage of cells [14\% of pyramidal neurons (Fig. 2Aiii); 17\% of interneurons (Fig. 2Aiv)] decreased their firing $(<0.5$ times) during ripples.

Specific discrepancies in discharge patterns between pyrami- 

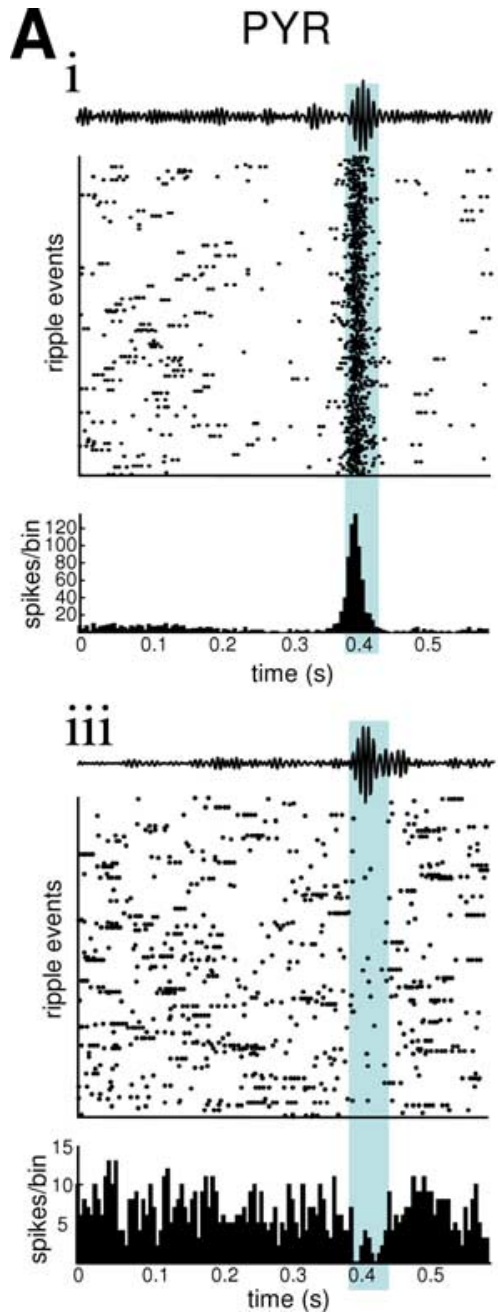
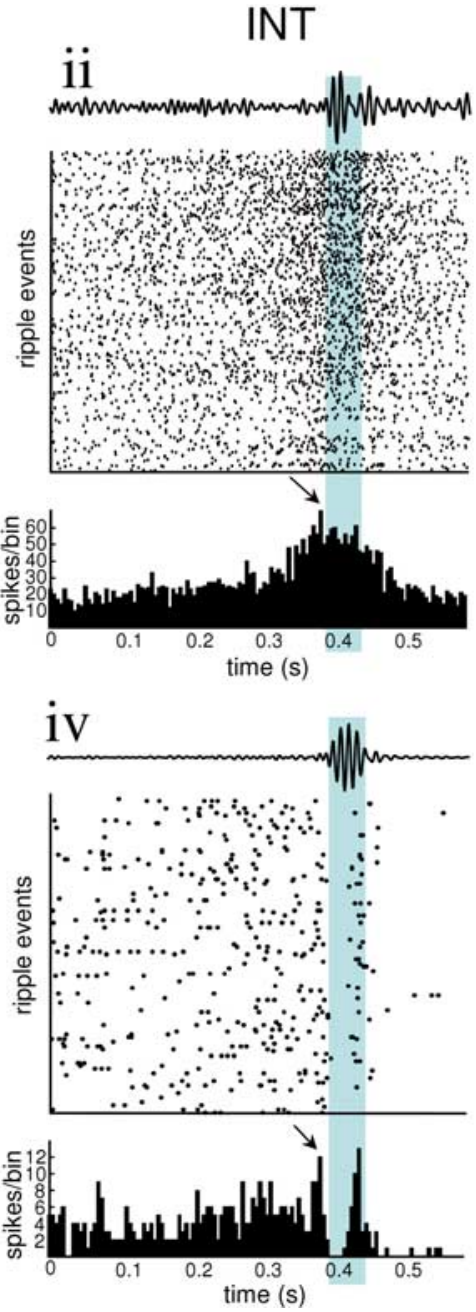

B
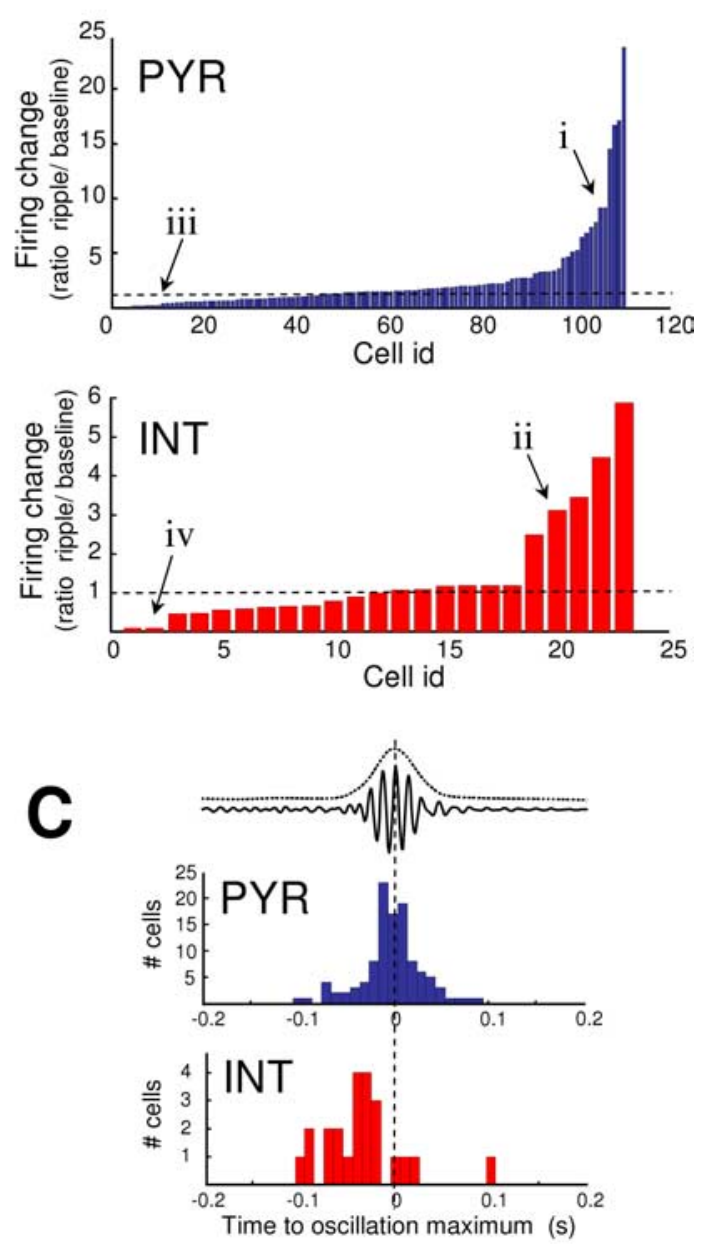

Figure 2. A, Ripple-triggered spike histograms (bin size, $5 \mathrm{~ms}$ ). Cells $\boldsymbol{i}$ and iii (ii and iv, respectively) are putative pyramidal cells (interneurons, respectively). Note that, for interneurons ii and iv, the maximal neuronal discharge (arrows) occurs before the field ripples. $\boldsymbol{B}$, Firing changes of interneurons (INT; red) and pyramidal cells (PYR; blue) during ripples, defined as the ratio between the discharge probability during ripples and baseline. C, Temporal relationship between the maximal neuronal discharge (red, interneurons; blue, pyramidal cells) and the highest peak of the oscillation envelope (dotted line).

dal cells and interneurons were observed during ripples in the rat (Ylinen et al., 1995; Csicsvari et al., 1999). Similarly, we found that, first, only pyramidal cells strongly increased (up to 24 -fold) their discharge probability during the ripple (Fig. $2 B$ ). In contrast, activated interneurons increased their firing by only twofold to fivefold relative to the baseline period (Fig. 2B). Second, pyramidal cell firing exhibits substantially more variability during ripples. Some neurons discharged during as much as $90 \%$ of the successive oscillations (Fig. $2 \mathrm{~A} i$ ), whereas other cells discharged very rarely $(<10 \%)$ (for typical examples, see supplemental Fig. S1 $i-i i$, available at www.jneurosci.org as supplemental material). In contrast, interneurons are more homogeneously engaged in the oscillations and often discharged during every successive ripple (on average, participation of $40 \%$ ). Third, as in rats (Csicsvari et al., 1999), on average, activated pyramidal cells fired maximally at the highest amplitude of the ripples (Fig. 2C). In contrast, interneurons discharged earlier, with maximum firing $50 \mathrm{~ms}$ before the peak of the ripple envelope (Fig. 2C). In six cases, interneurons increased their firing rate several hundreds of milliseconds before the ripple could be detected in the field potential (Fig. 2Aii) (for other examples, see supplemental Fig. S1iii-vi, available at www.jneurosci.org as supplemental material). In this group, two interneurons reached maximal firing before the ripple and then became silent during the ripple episode (Fig. 2Aiv) (supplemental Fig. S1vi, available at www. jneurosci.org as supplemental material). This pattern of activity closely resembles the firing behavior of axo-axonic interneurons during ripples reported in the rat hippocampus (Klausberger et al., 2003). Overall, these observations suggest similar roles for specific interneurons in structuring the activity of pyramidal cells (Somogyi and Klausberger, 2005).

In rodents, interneurons tend to fire after pyramidal cells during single-ripple cycles (Csicsvari et al., 1999; Buzsáki et al., 2003). To test this, we evaluated the phase values of spikes relative to the field oscillation (Jacobs et al., 2007). If the firing of a given neuron is independent of the ripple oscillation, the distribution of its phase values will be random. Conversely, if the firing of a given neuron is phase-locked to the ripples, its phase value distribution will be unimodal. The significance of this phase-locking was evaluated at $p=0.001$ by a Rayleigh's Z statistics (Fisher et al., 1993; Siapas et al., 2005). We found that $52 \%$ of the cells were significantly phase-locked to the ripple oscillations. This phenomenon was more pronounced than the changes in firing rate ( $\sim 25 \%$ of the neurons) described above and indicates that phase analysis provides additional information about the participation of neurons in the oscillations that cannot be measured by analyz- 

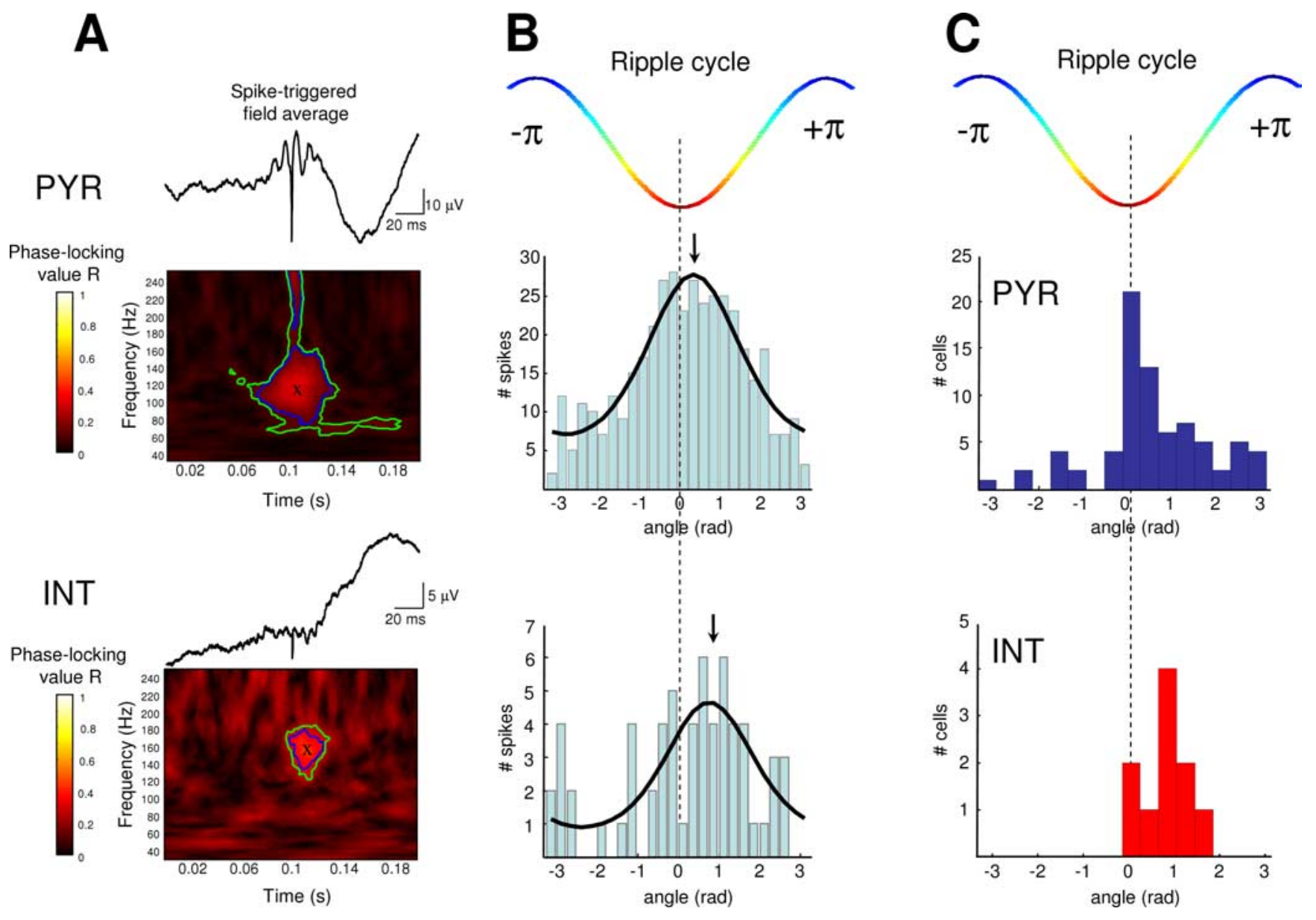

Figure 3. A, Phase-locking of spikes relative to the field ripple cycle (color panel; green contour line, $p<0.01$; blue contour line, $p<0.001$ ) for an interneuron (INT; bottom) and pyramidal cell (PYR; top). " $\mathrm{x}$ " indicates the frequency where phase locking is most statistically significant. The phase-locking of the cells is also confirmed by the spike-triggered field average (top black curve; average of 100 spikes), showing a strong high-frequency component of the averaged field. $\boldsymbol{B}$, Phase distribution of the spikes of the two cells illustrated in $\boldsymbol{A}$ (for the frequency indicated by " $\mathrm{x}$ " in A). Phase zero marks the trough of the ripple cycles. The distributions were fitted with a von Mises function (continuous black lines), and its mean gives the preferred firing phase (arrows). $\boldsymbol{C}$, Cumulative histogram of preferred firing phase for pyramidal cells (blue) and interneurons (red).

ing firing rate alone. Figure $3 A$ illustrates the phase-locking of a putative pyramidal neuron and an interneuron within the range of ripple frequencies. Phase-related firing was also confirmed by spike-triggered averages of the field during the oscillations (Fig. $3 A$ ). Furthermore, the distribution of their phases revealed that pyramidal cells fired preferentially during the troughs of the field ripples [mean firing phase, $0.5 \pm 0.9$ radians (rad)] (Fig. $3 B, C$ ). Interneurons (mean firing phase, $0.8 \pm 0.7 \mathrm{rad}$ ) fired on average $0.3 \mathrm{rad}$ later (corresponding to $\sim 0.5 \mathrm{~ms}$ for a $100 \mathrm{~Hz}$ ripple) (Fig. $3 B, C)$. These observations suggest a similar pyramidal cellinterneuron interaction during the ripple cycle as in rodents (Ylinen et al., 1995; Csicsvari et al., 1999).

\section{Discussion}

The present study explored the involvement of single cells during human ripples in vivo. We report the following observations: (1) a significant number of cells decrease or increase their firing rate during ripples, which suggests an active mechanism arises to selectively silence or enhance the activity of different neuronal groups; (2) pyramidal cells fired preferentially at the highest amplitude of the ripple, but interneurons began to discharge earlier than pyramidal cells; and (3) a large proportion of pyramidal neurons and interneurons are phase-locked to the ripple oscillations, but that the preferred phase of discharge of interneurons followed the maximum discharge probability of pyramidal neurons.

An important conclusion to be made from these analyses is that the relationship between field and electrophysiologically identifiable neuronal groups during ripples is qualitatively similar to that observed in vivo in rodents. This suggests that the physiological mechanisms that give rise to these patterns are similar. To date, two potential mechanisms have been advanced in rodents. One hypothesis proposes that ripples are brought about by a transient synchrony of interneuronal networks, excited by the CA3 input, which imposes a rhythmic inhibition onto pyramidal cells along the CA1-subicular-entorhinal axis (Ylinen et al., 1995; Csicsvari et al., 1999; Brunel and Wang, 2003; Buhl and Buzsáki, 2005). A second hypothesis is based on synchronization of pyramidal cells by gap junctions between their axons (Draguhn et al., 1998; Traub and Bibbig, 2000). Because interneurons increased their firing rate before pyramidal cells, and even before ripples, our findings favor the first hypothesis. The remarkably heterogeneous discharge patterns of putative interneurons during ripples provided additional clues to the possible synchronizing mechanisms. In particular, a subset of interneurons increase their firing before the ripples and then stop firing, similar to the pattern of firing of axo-axonic interneurons in rats that make 
inhibitory synapses at the site of action potential generation (Klausberger et al., 2003). Such firing patterns might inhibit pyramidal cells from firing at the beginning of the ripple episode but allow them to discharge at the highest amplitude of the oscillations. Other types of interneurons, driven later by pyramidal cell activity as suggested by their phases after the negative peak of the ripples, may limit the (potentially dangerous) increase in excitability within a local network. As basket cells or bistratified cells in the CA1 region of rats (Klausberger et al., 2003, 2004), these interneurons may control the fine tuning of spiking activity by providing a temporal structure relative to which the activity of individual pyramidal cells is coordinated. Such loops have been hypothesized previously to be important for the formation and retrieval of memories across cortical-hippocampal circuits (Buzsáki and Chrobak, 1995; Behrens et al., 2005). Therefore, our present observations could allow for a direct comparison of hippocampal data between rodents and primates, which opens the door to a broad across-species understanding of hippocampal function, including models of memory stabilization (Buzsáki, 1989; Lee and Wilson, 1992; Wilson and McNaughton, 1994). Furthermore, a morphological identification of recorded neurons is impossible in humans as it was done in animals, and finding similarities in the functions of neurons between human and animals opens the way to perform more adequate morphophysiological correlates in the human brain. In particular, the differential involvement of interneurons in fast oscillations could potentially be used to characterize different populations of interneurons in vivo in the human brain.

In addition, this understanding of the basic physiology of ripples is essential for describing the neural substrate of normal function as well as the pathological dysfunction of this substrate as manifest in epilepsy (Bragin et al., 1999, 2002a; Staba et al., 2002, 2004; Le Van Quyen et al., 2006). Ripple oscillations investigated in this study were recorded from patients with mesial temporal lobe epilepsy (MTLE) and can be considered normal electrographic phenomena. Ripple oscillations contrast with fast ripple oscillations, pathological oscillations of higher frequency (180-500 Hz) (Bragin et al., 1999, 2002a; Staba et al., 2002, 2004; Foffani et al., 2007). Fast ripples differ from ripples not only in their frequency range but by the fact that they can be recorded from dentate gyrus where normal ripples never occur, they appear to be localized exclusively in areas capable of generating spontaneous seizures, and they are generated by small discrete clusters of neurons, whereas generation of ripples is much more diffuse (Bragin et al., 2000, 2002b). Nevertheless, a recent study tracing the development of fast ripples after intrahippocampal kainate injection in rats documented the appearance of ripple frequency oscillations in dentate gyrus along with fast ripple frequency oscillations in this structure and elsewhere in hippocampus and parahippocampal regions (Bragin et al., 2004). It was concluded that the dentate ripple frequency oscillations were pathological markers of epileptogenesis, presumably reflecting the same mechanisms as fast ripples, rather than that of normal ripples. This finding raised the question as to whether at least some ripple frequency oscillations outside dentate gyrus in patients with MTLE and animal models of this disorder might also be pathological. In contrast to ripples, which are dependent on inhibitory mechanisms, fast ripples appear to reflect summated action potentials of bursting principal neurons (Bragin et al., $2002 b$ ). Because the population of ripples recorded in the present study were homogeneous with respect to underlying unit activity, it is reasonable to conclude that they all represent the same normal oscillatory mechanism and that pathological ripple fre- quency oscillations do not exist, or are very rare, in patients with longstanding MTLE. Pathological ripples could be a transient phenomena occurring in early stages of epileptogenesis during the development of fast ripples, or they may only exist in the dentate gyrus. The resolution of our ability to locate microelectrode tips in patients made it impossible for us to determine whether any of them may have actually been in the dentate gyrus. Whereas inhibitory mechanisms may be increased interictally in the epileptic hippocampus (Wilson et al., 1993, 1998), there is loss of paired-pulse suppression within discrete areas generating fast ripples (Bragin et al., 2002b). It is possible, therefore, that loss of critical inhibitory influences in these localized epileptogenic regions could mediate the transition of normal ripples to pathological fast ripples. Results of this study documenting neuronal activity underlying normal ripples are essential for future investigations into mechanisms of pathological fast ripples.

\section{References}

Behrens CJ, van den Boom LP, de Hoz L, Friedman A, Heinemann U (2005) Induction of sharp wave-ripple complexes in vitro and reorganization of hippocampal networks. Nat Neurosci 8:1560-1567.

Bragin A, Engel J, Wilson CL, Fried I, Buzsáki G (1999) High-frequency oscillations in human brain. Hippocampus 9:137-142.

Bragin A, Wilson CL, Engel J (2000) Chronic epileptogenesis requires development of a network of pathologically interconnected neuron clusters: a hypothesis. Epilepsia 41S:144-152.

Bragin A, Wilson CL, Staba RJ, Reddick M, Fried I, Engel J (2002a) Interictal high-frequency oscillations $(80-500 \mathrm{~Hz})$ in the human epileptic brain: entorhinal cortex. Ann Neurol 52:407-415.

Bragin A, Mody I, Wilson C, Engel J (2002b) Local generation of fast ripples in epileptic brain. J Neurosci 22:2012-2021.

Bragin A, Wilson CL, Almajano J, Mody I, Engel J (2004) High-frequency oscillations after status epilepticus: epileptogenesis and seizure genesis. Epilepsia 45:1017-1023.

Brunel N, Wang XJ (2003) What determines the frequency of fast network oscillations with irregular neural discharges? I. Synaptic dynamics and excitation-inhibition balance. J Neurophysiol 90:415-430.

Buhl DL, Buzsaki G (2005) Developmental emergence of hippocampal fastfield "ripple" oscillations in the behaving rat pups. Neuroscience 134:1423-1430.

Buzsáki G (1989) Two-stage model of memory-trace formation: a role for "noisy" brain states. Neuroscience 31:551-570.

Buzsáki G, Chrobak JJ (1995) Temporal structure in spatially organized neuronal ensembles: a role for interneuronal networks. Curr Opin Neurobiol 5:504-510.

Buzsáki G, Horvath Z, Urioste R, Hetke J, Wise K (1992) High-frequency network oscillation in the hippocampus. Science 256:1025-1027.

Buzsáki G, Buhl DL, Harris KD, Csicsvari J, Czeh B, Morozov A (2003) Hippocampal network patterns of activity in the mouse. Neuroscience 116:201-211.

Chrobak JJ, Buzsáki G (1996) High-frequency oscillations in the output networks of the hippocampal-entorhinal axis of the freely behaving rat. J Neurosci 16:3056-3066.

Chrobak JJ, Lorincz A, Buzsáki G (2000) Physiological patterns in the hippocampo-entorhinal cortex system. Hippocampus 10:457-465.

Clemens Z, Mölle M, Eross L, Barsi P, Halász P, Born J (2007) Temporal coupling of parahippocampal ripples, sleep spindles and slow oscillations in humans. Brain 130:2868-2878.

Csicsvari J, Hirase H, Czurko A, Mamiya A, Buzsáki G (1999) Oscillatory coupling of hippocampal pyramidal cells and interneurons in the behaving rat. J Neurosci 19:274-287.

Csicsvari J, Hirase H, Mamiya A, Buzsáki G (2000) Ensemble patterns of hippocampal CA3-CA1 neurons during sharp wave associated population events. Neuron 28:585-594.

Draguhn A, Traub RD, Schmitz D, Jefferys JG (1998) Electrical coupling underlies high-frequency oscillations in the hippocampus in vitro. Nature 394:189-192.

Fisher N (1993) Statistical analysis of circular data. Cambridge: Cambridge UP.

Foffani G, Uzcategui YG, Gal B, Menendez de la Prida L (2007) Reduced 
spike-timing reliability correlates with the emergence of fast ripples in the rat epileptic hippocampus. Neuron 55:930-941.

Fried I, Wilson C, Maidment N, Engel J, Behnke E, Fields T, MacDonald K, Morrow J, Ackerson L (1999) Cerebral microdialysis combined with single-neuron and electroencephalographic recording in neurosurgical patients. J Neurosurg 91:697-705.

Harris KD, Hirase H, Leinekugel X, Henze DA, Buzsáki G (2001) Temporal interaction between single spikes and complex spike bursts in hippocampal pyramidal cells. Neuron 32:141-149.

Hartigan JA, Hartigan PM (1985) The dip test of unimodality. Ann Statistics 13:70-84.

Hazan L, Zugaro M, Buzsáki G (2006) Klusters, NeuroScope, NDManager: a free software suite for neurophysiological data processing and visualization. J Neurosci Methods 155:207-216.

Jacobs J, Kahana MJ, Ekstrom AD, Fried I (2007) Brain oscillations control timing of single-neuron activity in humans. J Neurosci 27:3839-3844.

Klausberger T, Magill PJ, Marton LF, Roberts JD, Cobden PM, Buzsaki G, Somogyi P (2003) Brain-state- and cell-type-specific firing of hippocampal interneurons in vivo. Nature 421:844-848.

Klausberger T, Marton LF, Baude A, Roberts JD, Magill PJ, Somogyi P (2004) Spike timing of dendrite-targeting bistratified cells during hippocampal network oscillations in vivo. Nat Neurosci 7:41-47.

Lee AK, Wilson MA (2002) Memory of sequential experience in the hippocampus during slow wave sleep. Neuron 36:1183-1194.

Le Van Quyen M, Bragin A (2007) Analysis of dynamic brain oscillations: methodological advances. Trends Neurosci 30:365-373.

Le Van Quyen M, Khalilov I, Ben-Ari Y (2006) The dark side of highfrequency oscillations in the developing brain. Trends Neurosci 29:419-427.

Mechler F, Ringach DL (2002) On the classification of simple and complex cells. Vision Res 42:1017-1033.

O’Keefe J, Nadel L (1978) The hippocampus as a cognitive map. Oxford: Oxford UP.

Siapas AG, Lubenov E, Wilson MA (2005) Prefrontal phase-locking to hippocampal theta oscillations. Neuron 46:141-151.
Skaggs WE, McNaughton BL, Permenter M, Archibeque M, Vogt J, Amaral DG, Barnes CA (2007) EEG sharp waves and sparse ensemble unit activity in the macaque hippocampus. J Neurophysiol 98:898-910.

Somogyi P, Klausberger T (2005) Defined types of cortical interneurone structure space and spike timing in the hippocampus. J Physiol (Lond) 562:9-26.

Staba RJ, Wilson CL, Bragin A, Fried I, Engel J (2002) Quantitative analysis of high-frequency oscillations $(80-500 \mathrm{~Hz})$ recorded in human epileptic hippocampus and entorhinal cortex. J Physiol (Lond) 88:1743-1752.

Staba RJ, Wilson CL, Bragin A, Jhung D, Fried I, Engel J (2004) Highfrequency oscillations recorded in human medial temporal lobe during sleep. Ann Neurol 56:108-115.

Traub RD, Bibbig A (2000) A model of high-frequency ripples in the hippocampus, based on synaptic coupling plus axon-axon gap junctions between pyramidal neurons. J Neurosci 20:2086-2093.

Urrestarazu E, Chander R, Dubeau F, Gotman J (2007) Interictal highfrequency oscillations $(100-500 \mathrm{~Hz})$ in the intracerebral EEG of epileptic patients. Brain 130:2354-2366.

Viskonsas IV, Ekstrom AD, Wilson CL, Fried I (2007) Characterizing interneuron and pyramidal cells in the human medial temporal lobe in vivo using extracellular recordings. Hippocampus 17:49-57.

Wilson CL, Engel J (1993) Electrical stimulation of the human epileptic limbic cortex. In: Electrical and magnetic stimulation of the brain and spinal cord (Devinsky O, Berie A, Dogali M, eds), pp103-113. New York: Raven.

Wilson CL, Khan SU, Engel J, Isokawa M, Babb TL, Behnke EJ (1998) Paired pulse suppression and facilitation in human epileptogenic hippocampal formation. Epilepsy Res 31:211-230.

Wilson MA, McNaughton BL (1994) Reactivation of hippocampal ensemble memories during sleep. Science 265:676-679.

Ylinen A, Bragin A, Nadasdy Z, Jando G, Szabo I, Sik A, Buzsáki G (1995) Sharp wave-associated high-frequency oscillation $(200 \mathrm{~Hz})$ in the intact hippocampus: network and intracellular mechanisms. J Neurosci 15:3046. 